\title{
The Asymmetric Cosmic Time - The Key to a New Cosmological Model
}

\author{
Horst Fritsch $^{1, *}$ and Eberhard Schluecker ${ }^{2}$ \\ ${ }^{1}$ Retired scientist, D-71229 Leonberg, Germany \\ ${ }^{2}$ Institute for systems engineering, University of Erlangen- Nuremberg, D-91058 Erlangen, Germany
}

\section{Article Info}

*Corresponding author:
Horst Fritsch
Retired scientist
D-71229 Leonberg
Germany
E-mail: fritsch-leonberg@t-online.de

Received: March 16, 2020

Accepted: August 25, 2020

Published: August 31, 2020

Citation: Fritsch $\mathrm{H}$, Schluecker E. The Asymmetric Cosmic Time - The Key to a New Cosmological Model. Int J Cosmol Astron Astrophys. 2020; 2(1): 97-111. doi: 10.18689/ijcaa-1000122

\section{Copyright: @ 2020 The Author(s). This work is licensed under a Creative Commons Attribution 4.0 International License, which permits unrestricted use, distribution, and reproduction in any medium, provided the original work is properly cited.}

Published by Madridge Publishers

\section{Abstract}

The asymmetric cosmic time is a logical consequence of the General Theory of Relativity (GR), if one demands that it should apply to the entire cosmos.

From the simplest cosmological model that is consistent with the ART (Einstein-de Sitter model) thus follows the < Cosmic Time Hypothesis $>(\mathrm{CTH})$, which offers solutions for many unsolved problems of cosmology that the current standard model of cosmology ( $\Lambda \mathrm{CDM}$ model) cannot explain.

According to the $\mathrm{CTH}$, space, time and matter form a unit and develop evolutionarily according to identical, time-dependent laws. According to the CTH time has neither beginning nor end. The "big bang" disappears into the infinite past, which is why the universe manages without inflation. The accelerated expansion of the universe is also unlikely to occur if the $\mathrm{SN}$-la measurement results are interpreted using the $\mathrm{CTH}$. The cosmological constant $\Lambda$ can then be omitted $(\Lambda=0)$ and consequently no "dark energy" is needed.

In addition, the $\mathrm{CTH}$ also provides interesting results on the topics: Initial conditions for hypotheses, stability of the expanding, flat universe $(\Omega=1)$, cosmic energy balance (is there negative energy ?), theory of earth expansion, unification of natural forces, Mach's principle.

Should the CTH receive broad experimental confirmation, the GR could be extended to the "Universal Relativity Theory" (UR).

Keywords: Cosmic Time Hypothesis; Hubble-time; Quantum field theory; General relativity theory; Gravitational constance; Newtonian time; Gravitational force; Planck's time.

Abbreviations: CTH: Cosmological Time Hypothesis; GR: General theory of Relativity; GTR: General Relativity Theory; $\Lambda$ CDM: Current Standard Model of Cosmology; QFT: Quantum Field Theory; SNla: Supernovae type la; SRT: Relative movements in Space.

\section{Introduction}

The <Cosmic Time Hypothesis $>$ (CTH) presented in this paper is an alternative to the current standard model of cosmology ( $\Lambda C D M$ model). It argues for a real existing time, which interprets the whole universe as an evolutionary evolving system. Thus, it stands in strong contrast to the "block universe" propagated today, in which time is only an illusion. The CTH is not only simpler and more consistent than the $\Lambda$ CDM model, but also has a much higher explanatory potential.

In advance their most important results:

- It solves one of the biggest problems of cosmology - the problem of the cosmological 
constant $(\Lambda)$ - by abolishing the relationship between $\Lambda$ and the vacuum energy density $\left(\Lambda=0, \varepsilon_{\mathrm{v}}>0\right)$. According to the $\mathrm{CTH}$, the vacuum energy density $v$ is not negative and constant, as previously assumed, but positive and timedependent $\left(\varepsilon_{\mathrm{v}} \sim t^{-2}\right) . \varepsilon_{\mathrm{v}}$ is part of the total energy density $(\varepsilon)$ of the universe and is contained in the energy pulse tensor of Einstein's field equations. The cosmology is thus freed from unnecessary ballast, i.e. a free parameter ( $=$ natural constant $)$ is omitted $(\Lambda=0)$. Conclusion: There is no "dark energy"!

- According to the $\mathrm{CTH}$, the numerical value of the vacuum energy density $v$ is smaller by a factor of $\approx 10^{-122}$ than the value calculated from quantum field theory and is therefore in accordance with the observation.

- The measured data obtained from observations of SNla supernovae, which suggest a currently accelerated expansion of the universe, result - if interpreted from the point of view of the CTH - in a slowed expansion, as required by the Einstein-de Sitter universe.

- The "dark matter" could also possibly not exist, because the CTH demands that the "gravitational constant" is time-dependent and becomes larger, the further away the observed objects are from us in space and time.

- Gravitatively bound local systems e.g. earth - moon or sun - earth expand according to the same law as the universe. This explains why Hubbles' law also applies within very small groups of galaxies, as observations show.

- The CTH demands that the strongest force (strong nuclear force) and the weakest (gravitational force) at Planck's time $\left(t_{p} \approx 10^{-43}\right.$ seconds after the "Big Bang") when all forces of nature were supposed to have been united in a single superpower, were of equal size and had the same range. According to the $\mathrm{CTH}$, the product of the strength and range of the gravitational force is constant, i.e. independent of time, and is identical to the product of the strength and range of the strong nuclear force.

- At Planck's time, the universe had the size of an elementary particle $\left(R_{p}=r_{E} \approx 10^{-15} \mathrm{~m}\right)$. This value also corresponds to the range of the strong nuclear force (Yukawa radius) and the Planck length at Planck time.

- The CTH provides a possible explanation for Mach's first and second principles.

- It solves some old problems of the Big Bang theory in a simple and natural way. The problem of the horizon, the flatness, the formation of galaxies and the age of the world. The theory of inflation becomes superfluous.

- The CTH provides the theoretical basis for the theory of earth expansion.

- In Cosmic Time there was no Big Bang. The universe is infinitely old.

- In contrast to other cosmological models, the CTH does not need defined "initial conditions" because there was no beginning.
- From the point of view of the $\mathrm{CTH}$, Einstein's field equations are followed by an evolutionary universe evolving in cosmic time.

- The CTH explains why cosmic expansion is permanently in the unstable state of equilibrium necessary for a longterm flat (Euclidean) evolutionary universe.

As shown below, the CTH is compatible with the General Theory of Relativity (GTR). However, it increases its significance by introducing cosmic time.

\section{The Cosmic Time Hypothesis (CTH) - a New Interpretation of the Einstein-de Sitter Universe}

For the Einstein-de Sitter universe, Einstein has formulated the relationship [1], p. 117:

$$
\frac{x e}{3}-h^{2}=0
$$

$\left(\mathcal{u}=8 \pi \mathrm{G} / \mathrm{c}^{2}\right.$ : coupling constant of Einstein's field equations, G: gravitational constant, $c$ : vacuum speed of light, $\rho=M /$ $\mathrm{V}=3 \mathrm{M} / 4 \pi \mathrm{R}^{3}$ : mean mass density of the universe, $R$ : cosmic radius, $\mathrm{h}=1 / c t_{H}=1 / R, t_{H}$ : Hubble time)

By transforming equation (1) one obtains

$$
\frac{G M}{R c^{2}}=\frac{1}{2}
$$

This equation contradicts the current state of knowledge, according to which $G, M$ and $c$ are constant, R- however, increases with time (in the Einstein-de Sitter universe: $R \sim t^{2 / 3}$ ).

It is interesting to note that as early as 1917 , shortly after the publication of the GTR, Einstein formulated an almost identical relationship for the model cosmos named after him, similar to equation (2) [2].

$$
\frac{G M}{R c^{2}} \approx 1
$$

Unfortunately, it was forgotten again after Edwin Hubble had experimentally demonstrated that the universe cannot be static, which Einstein originally assumed, but is expanding.

The following prerequisites (axioms) shall apply for the further explanations:

I. The speed of light is a universal natural constant

II. The cosmological constant has a numerical value of zero $(\Lambda=0)$.

III. Averaged over long distances, space is flat $(\Omega=1)$.

IV. The Universe is homogeneous and isotropic on large scales.

V. The Universe Expands at the Speed of Light $(\dot{R}=c)$.

VI Total energy in the universe is constant.

VII. The law of entropy increase is fundamental.

With the plausible assumption that space is flat $(\Lambda=0)$ and expands at the speed of light (Axiom $V$ ), the velocity of light results with:

$$
R \sim t^{2 / 3}
$$


to this equation:

$$
c=\dot{R}=\frac{d R}{d t} \sim t^{-1 / 3}
$$

This contradicts the postulate $c=$ constant $($ Axiom I)

So we have to clarify: What do we understand by the term "natural constant"?

Answer: Natural constants are physical quantities that can only be determined empirically and cannot be derived from a superordinate theory. The statement $c=$ constant thus means that the measured numerical value of $c$ must always be the same at any place and at any time. The problem now is to bring this requirement into agreement with the relation Eq (5).

Einstein had similar difficulties with the development of the special theory of relativity (SRT), by the way, until shortly before its publication he found the solution which he enthusiastically told his friend Michele Besso one morning [3]: "... I solved the problem completely. My solution was an analysis of the concept of time. Time cannot be defined absolutely and there is an irreversible relationship between time and signal velocity".

Later he formulated this even more concretely [1]: "One can use the principle of the constancy of the vacuum speed of light to complete the definition of time". Could it be that time has to be relativized again, so that the axiom c=constant is valid for all events in space-time"?

With the assumption that we measure the speed of light as the greatest possible signal velocity, not only with relative movements in space (SRT) and different gravitational fields (GTR) as a constant quantity, but also at different times, the demand for a further flexibilization of the concept of time results from equation (5). The task is therefore to find a time measure that measures the speed of light as a constant value at all times. This results, if one introduces a time $\tau$, which changes proportional to the world radius. An idea, by the way, which Henning Genz already had [4], p. 229: "Then why not go all the way and choose the radius of the universe as time parameter"?

For the Einstein de Sitter universe one then obtains for this cosmic time $\tau$ the relationship

$$
\tau \sim R \sim t^{2 / 3}
$$

and

$$
\frac{d \tau}{d t} \approx \frac{\Delta \tau}{\Delta t} \sim t^{-1 / 3} \sim \dot{\mathrm{R}}=c
$$

In fact, as will be shown later, pendulum clocks and atomic clocks indicate exactly this cosmic time when they tick according to the laws of the CTH (see Appendix "Rate speed of watches according to CTH"). Measured with such clocks, the speed of light is then a constant quantity:

$$
c(\tau)=d R / d \tau=\text { constant }
$$

Alexander Friedmann would certainly have agreed with this approach. In his book <The World as Space and Time>, published in Petrograd at the beginning of 1923, he wrote [5], p. 73: "(Abstract) time can be arithmetized in a completely arbitrary manner; each moment will always correspond to a certain number $t$. The transition from an arithmetization of the number considered alone to another is expressed by replacing the number $t$ with $\tau=f(t)$.... Then $d \tau=T d t$, where $T$ is dependent on $t^{\prime \prime}$.

Exactly this dependence contains the relation (7).

$$
d \tau=K \cdot t^{-1 / 3} \cdot d t\left(K \cdot t^{-1 / 3}=T, K=\text { constant }\right)
$$

Henning Genz could also have agreed to this [4], p. 225: "The laws of general relativity are independent of the parameterization of time".

Thus the time cycle would not only depend on the relative velocity (SRT) and the gravitational potential (GTR), but also on the time itself (CTH). A comparison of these dependencies is shown in figure 1.

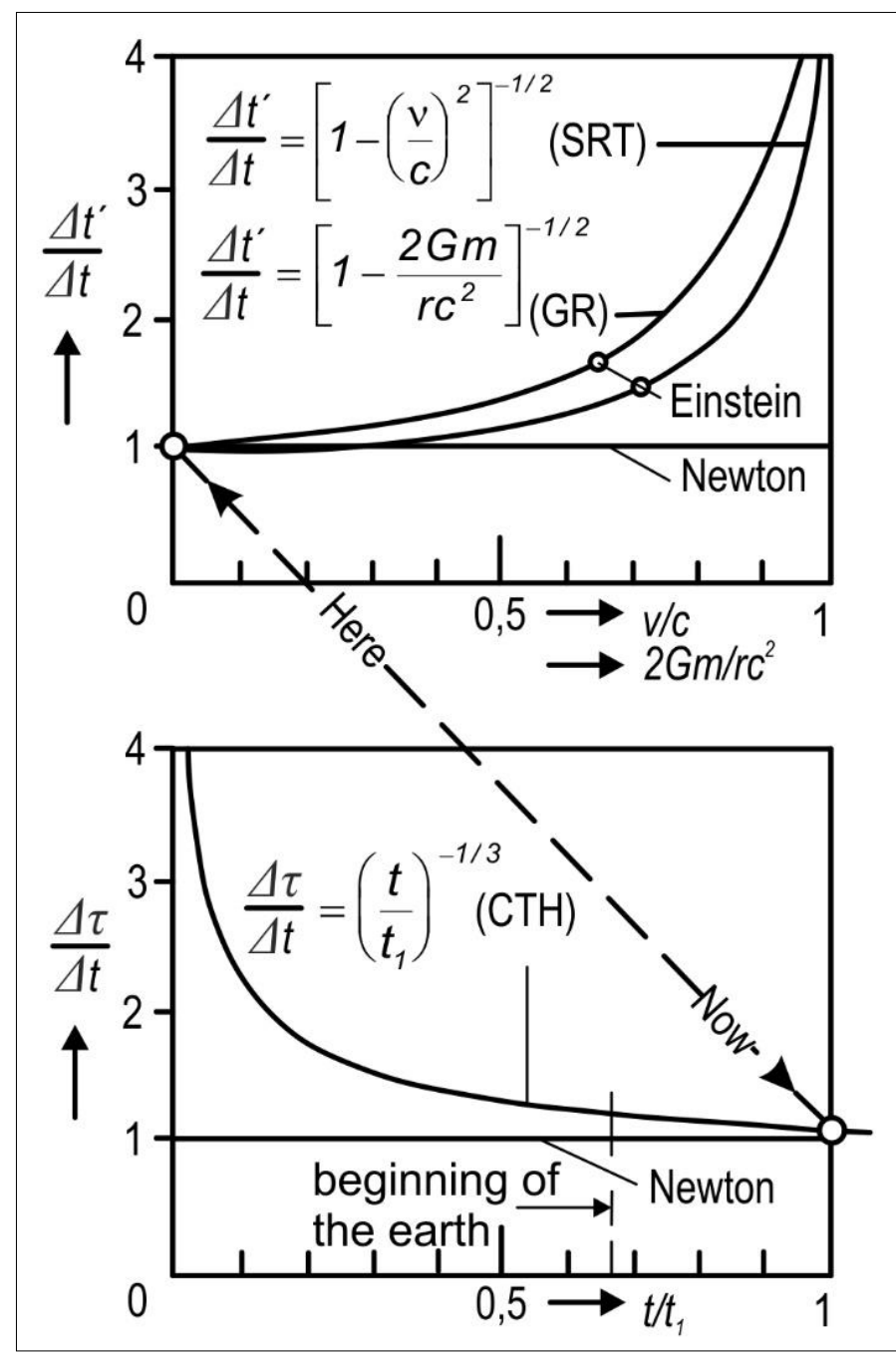

Figure 1. Time measurements of SRT, GR and CTH.

The GTR thus forces us to introduce cosmic time $\tau$ in order to bring it into agreement with the equation $\mathrm{GM} / \mathrm{Rc}^{2}=1 / 2$ derived from it. In plain language this means: The GTR is time asymmetric! It has a cosmological time arrow and thus follows the 2, main theorem of thermodynamics (Axiom VII).

This means that the total cosmic entropy in the expanding 
universe (=closed system) is constantly increasing. Within the system, however, a local decrease in entropy can occur if it is compensated by the increase in entropy of the environment.

The following applies to the universe: The unlikely original state of a continuous, structureless energy distribution is transformed by gravity into the more probable state of a structured energy (mass) distribution. Here is a quote from Brian Greene [6]:

There is convincing evidence for this and little doubt that in the early history of the universe, matter was uniformly distributed throughout space. Usually this would be described as a highly entropic configuration - such as the carbon dioxide molecules from a coke bottle that were evenly distributed throughout a room - then it would be a commonplace and would not require explanation. But if gravity plays a role, as it does when we look at the entire universe, then a uniform distribution of matter is a rare, low-entropic, highly ordered configuration, because gravity causes matter to form lumps. Correspondingly, a smooth and uniform curvature of space also has a low entropy. It is highly ordered in comparison to an extremely uneven, non-uniform curvature of space.

From the relations (2), (4) and (5) one obtains.

$$
G M=\text { constant }
$$

Based on the assumption that the overall energy $E$ in the universe is constant (Axiom $\mathrm{VI})$, results from $\mathrm{E}=\mathrm{Mc}^{2}$ and (5) for the mass of the universe.

$$
M \sim t^{2 / 3} \sim R
$$

In this, $\mathrm{M}$ means the total gravitatively effective energy existing in the universe $\left(\mathrm{M}=\mathrm{E} / \mathrm{c}^{2}\right)$. In addition to the ponderable mass, this also includes the radiation and vacuum energy. All these forms of energy are, as will be shown later, positive and contained in the energy pulse tensor of Einstein's field equations. The further relations result from (4), (9) and (10).

Gravitation constant:

$$
G \sim M^{-1} \sim t^{-2 / 3}
$$

Mean mass density of the universe:

$$
\varrho \sim R^{-2} \sim t^{-4 / 3}
$$

Mean energy density of the universe:

$$
\varepsilon=\varrho c^{2} \sim t^{-2}
$$

Figure 2 summarises the results to date.

One could now object that time-varying "natural constants" ( $\left(\sim t^{-1 / 3}, G \sim t^{-2 / 3}\right)$ are not compatible with the GTR. But since $c$ and $G$ do not appear solitary in the field equations, but are linked by the coupling constant $x=8 \pi G / c^{2}$, there is no contradiction between GTR and CTH.

The value $\mathrm{G} / \mathrm{c}^{2}$ is time-independent and amounts to 7,41 $\cdot 10^{-28} \mathrm{~m} / \mathrm{kg}$.

\section{The labile equilibrium of cosmic expansion}

By conventional view, universe expanding by equation (2) would be unstable. Cosmic expansion could only remain in unstable equilibrium, if besides $R(t)$ also other quantities in equation (2) are time- dependent, like demanded by CTH (Figure 2).

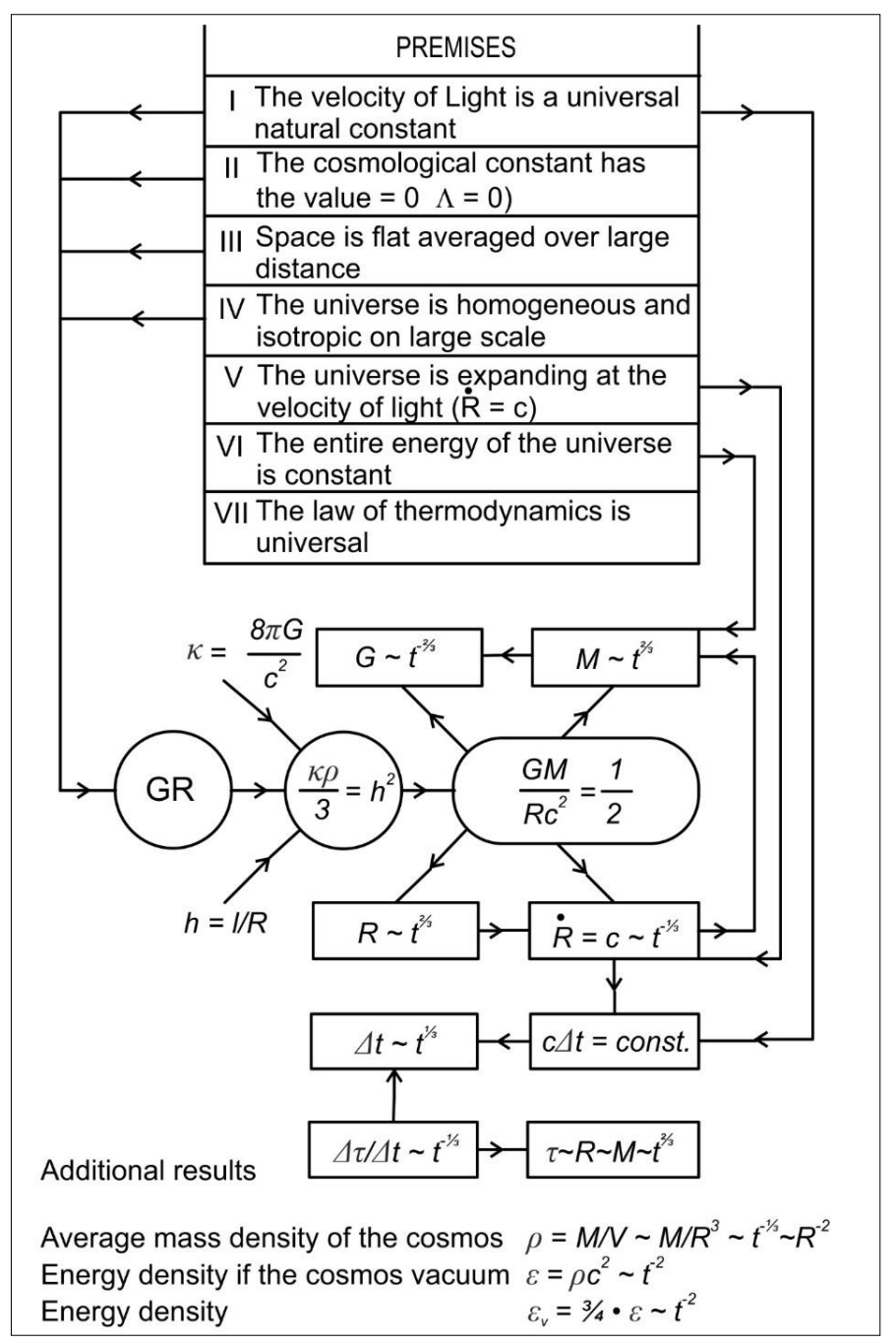

Figure 2. Summary of the results (2) up to (13).

Realistically, one has to imagine the expansion of the universe as a process that is open to the future and evolves over time, generating new, emergent manifestations again and again. Such a process cannot be reconciled with the timesymmetric block universe.

Ilya Prigogine [7] and Lee Smolin [8] see a fundamental problem for the entire natural sciences in the contradiction between time symmetry (block universe), in which time is reversible and is therefore regarded as an illusion, and time asymmetry, which gives time a direction.

Prigogine demands that the second law of thermodynamics should be as general as the postulate $c=$ constant. For cosmology this necessarily leads to asymmetric time, because it is necessary to keep cosmic expansion in unstable equilibrium in the long run.

How the permanent oscillation into the equilibrium state takes place will be explained briefly.

According to the $\mathrm{CTH}: c \sim t^{-1 / 3} \mathrm{G} \sim t^{-2 / 3}, \mathrm{G} / \mathrm{c}^{2}=$ constant. 
Case I: Fluctuations cause the universe to expand somewhat faster than eq. (5), i.e. $\dot{R}_{\text {def }}>\dot{R}_{\text {target }}$ Because $\dot{R}=c$ and $G \sim C^{2}$ is $G_{\text {def }}>G_{\text {target }}$ which delays expansion.

Case II: The universe expands slightly slower than eq. (5), i.e. $\dot{R}_{\text {def }}<\dot{R}_{\text {target }}$ Then $G_{\text {def }}$ is $<G_{\text {targett }}$ which accelerates expansion.

Seen in this light, the universe can be compared to a tightrope walker who keeps his balance through small changes in his posture. So the universe exists only because the asymmetry of time forces the cosmic expansion to constantly settle into the unstable state of equilibrium of a flat universe.

\section{Cosmological energy balance}

Many cosmologists, such as St. Hawking, L.M. Krauss, H.J. Fahr and others, assume that the total energy in the universe is exactly zero. They postulate a negative gravitational binding energy that exactly balances the positive energy (matter and radiation) [9], p. 164: "For a universe that is largely uniform in spatial terms, one can prove that this negative gravitational energy cancels exactly the positive energy represented by matter. Therefore, the total energy of the universe is zero".

And further: "If the size of the universe doubles, the positive matter energy and the negative gravitational energy also doubles, so that the total amount remains zero".

Here it was determined that the potential energy between two bodies is zero if they are infinitely far from each other [10]. S. 137: "It is negative (the gravitational energy), because we define the total gravitational energy of an object infinitely distant from any other object at rest as zero".

This zero point definition for the gravitational energy is, in my opinion, inadmissible, since the greater the distance between two masses, the greater the potential (positive) energy between them, and it reaches a maximum when the distance becomes infinite.

Why one then defines this energy state as zero, is difficult to understand, because it corresponds exactly to the kinetic energy, which is in the escape velocity, which is necessary, in order to bring two masses on infinitely large distance.

That the gravitational energy of an object at rest, which is infinitely far away from all other objects, should have the amount zero, is furthermore not compatible with Hawking's statement (see quote above) that with doubling of the size of the universe both the positive matter energy and the negative gravitational energy double, so that the total energy always remains zero.

Some scientists, including Paul Davies, explain negative gravitational energy by saying that energy must be used to remove two masses from the gravitational field connecting them [11], pp. 66-68: "If we were to try to tear the earth out of its orbit around the Sun, we would have to do a lot of hard work, i.e. apply energy to arrive against the attraction of the Sun. But this means that the gravitational energy that binds the earth to the Sun is negative, since work is needed to release the bond. If, however, the gravitational field has a negative energy, it must also have a negative mass, which must then be subtracted from the positive mass energy of the sun and the planets".

Here it is concealed that the planets orbiting the sun possess kinetic energy, which - as the calculation shows (see Appendix "Kinetic and potential energy of planets") corresponds quite exactly to that which would have to be used to bring them from their present position to astronomically great distance. Conversely, an object far away from a large mass and at rest, gravitatively attracted by that mass and finally orbiting, would have the same kinetic energy as it previously had as potential energy.

So, in terms of amount, both forms of energy are equal, but they differ in their entropy. The Sun-Earth system has a higher entropy than it would be if both celestial bodies were very far away. In order to free them from their bond, i.e. to bring them into a state of lower entropy, the second law of thermodynamics states that energy coming from outside (outside the system) must be supplied.

An example from chemistry (Figure 3 ) should serve as an illustration. When hydrogen $(\mathrm{H})$ and oxygen $(\mathrm{O})$ react chemically, water $\left(\mathrm{H}_{2} \mathrm{O}\right)$ and heat are produced, whereby the total energy for both states is retained, but entropy increases. The thermal energy generated during the reaction is called binding energy, which must be used to release the binding again.

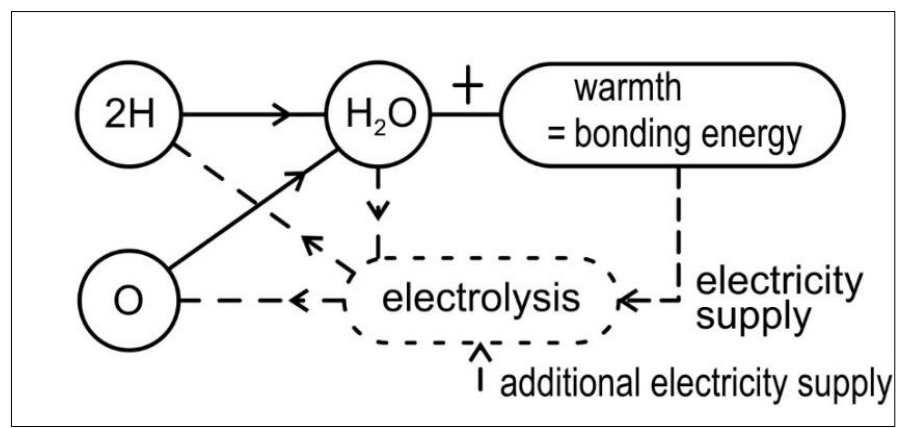

Figure 3. Irreversible chemical process.

Purely theoretically, if the reaction heat is fully exhausted, water could be broken down into hydrogen and oxygen (electrolysis). However, the 2nd law of thermodynamics prohibits the realization of this process! Only when additional energy is supplied from outside can the water be completely broken down into hydrogen and oxygen again. Also in many other "binding processes" (gravitational bonds, chemical reactions, nuclear fusions) lower entropic energy is converted into higher entropic energy. However, it remains a mystery why the term binding energy is then assigned a negative sign.

In my opinion, negative energy is a purely theoretical construct, which follows from the (arbitrary) determination of the energy zero point. In physical reality there is no negative energy! By the way, Einstein also dealt with the problem of "negative energy" after he had banished the cosmological constant $\Lambda$, which was originally introduced by himself and can be understood as a synonym for negative energy or negative pressure, from his field equations. The result of his considerations was [1], p. 110: "The questionable thing about 
this solution (field equations with $\Lambda>0$ ) is that one has to introduce a negative pressure for which there is no physical justification". So he was also of the opinion that negative energy should not exist.

\section{Space and time perspectives}

If we project objects in (3-dimensional) space onto a (2-dimensional) plane, such as in a photograph, the further away they are from the place where they were taken, the smaller they become. With an "infinitely" long tree avenue, whose trees are all of the same size and have the same distance from each other, very distant objects focus in the central perspective (Figure 4) in the so-called vanishing point. Since we can move freely in space, we know from experience which size distant objects really are.

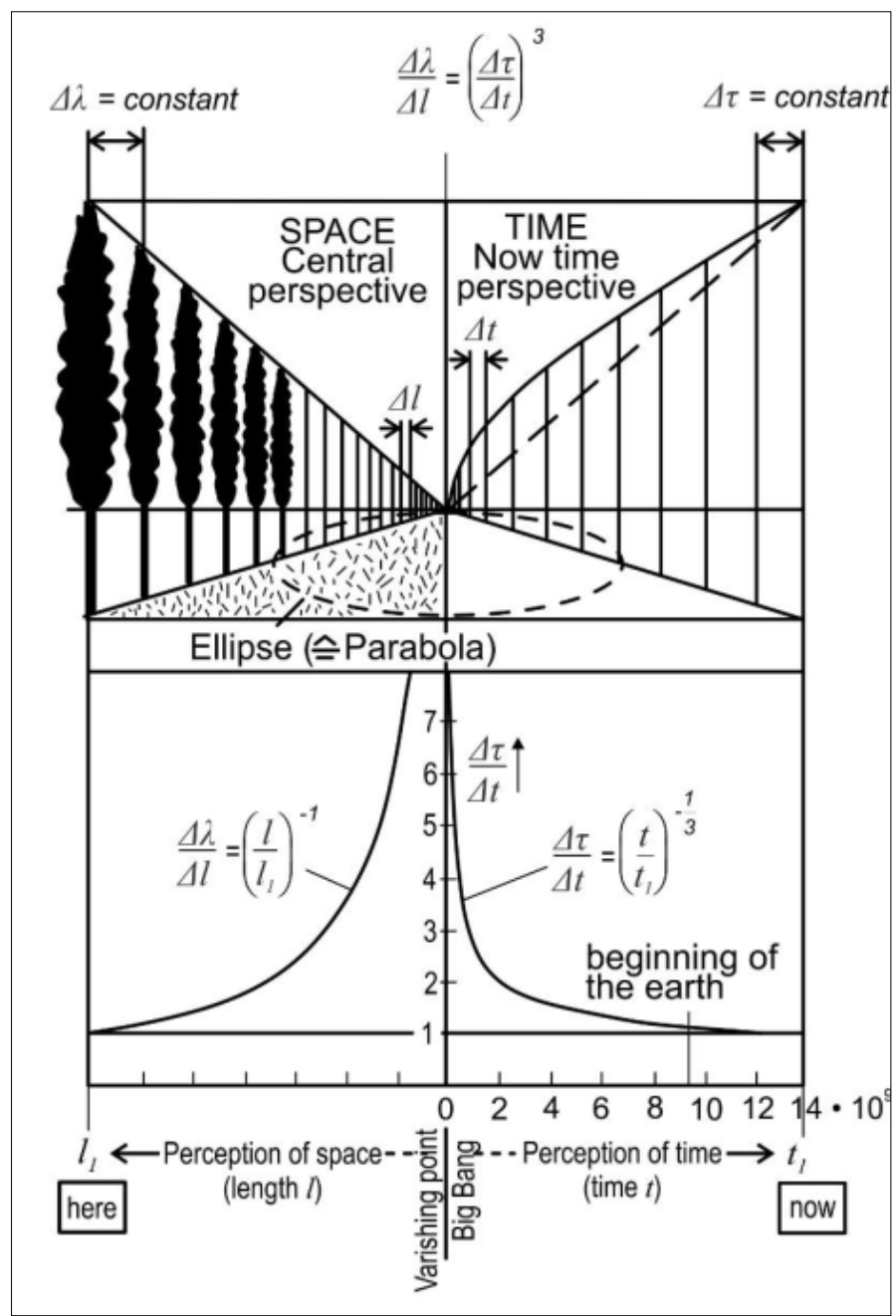

Figure 4. Space and time truth in comparison.

In time, on the other hand, we are trapped in the "now" and have no way of checking how the time clock was outside the "now". According to the CTH it must have been shorter in the past than today (Figure 4), i.e. the clocks must have ticked faster from "today view", in the "Big Bang" even infinitely fast $(\Delta t=0)$. That would move the Big Bang singularity into the infinitely distant past, [12].

It is also interesting when comparing space and time (Figure 4) that the "perspective shortenings" of 1- dimensional time and 3-dimensional space correspond to the relationship $\Delta \lambda / \Delta \mathrm{l}=(\Delta \tau / \Delta t)^{3}$.
Figure 4 shows that the time interval $\Delta \mathrm{t}$ is dramatically shortened only very close to the Big Bang singularity, i.e. the universe expanded, related to present time, shortly after the "Big Bang" at extremely high speed, but not inflationary, as required by inflation theory. From the point of view of the cosmic time $\tau$, however, it expanded at the same speed as it does today, at a "constant" speed of light $(c(\tau)=d R /$ $d \tau=$ constant). Currently, the time interval $\Delta$ t changes only marginally, namely only by $3.5 \cdot 10^{-11} /$ years, which is about one millisecond per year. Even 4.6 billion years ago, when our solar system was formed, the clocks ran just $14 \%$ faster than today (Figure 4).

\section{The unity of space, time and matter}

A surprising and also aesthetically impressive result of the CTH is that space $(R)$, cosmic time $(\tau)$ and matter $(\mathrm{M})$ develop according to identical laws $(R \sim \tau \sim M)$.

The physical basic concepts - space, time and matter with which we describe our perceptible reality, are thus closely interlinked.

In comparison to the $\mathrm{CTH}$, the $\Lambda$ CDM model lacks a harmonious connection between the basic parameters of cosmology. The $\Lambda$ CDM model states that after a complicated time function $(R=R(t))$ the universe today expands at an accelerated rate, but time progresses continuously linearly $(\mathrm{t}=\mathrm{t})$ and matter has remained constant since its formation in the early universe and will continue to remain constant (M=constant).

Figure 5 shows the comparison between $\mathrm{CTH}$ and the $\Lambda$ CDM model.

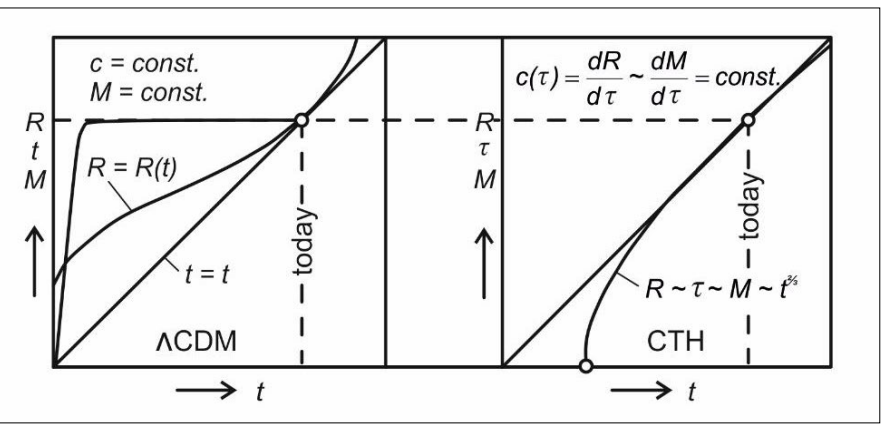

Figure 5. $\triangle \mathrm{CDM}$ model and $\mathrm{CTH}$ model compared.

\section{What the CTH does}

\section{Solving old big bang theory problems (horizon, flatness, galaxy formation and age problem)}

For all these problems, the $\Lambda$ CDM model suggests solutions by means of some ad hoc hypotheses (inflation, dark matter, dark energy), but they are questionable because they do not legitimize themselves convincingly. On the other hand, the CTH offers much more plausible solutions.

As figure 6 shows, it solves the problem of the horizon by means of the time-variable speed of light $\left(c \sim t^{-1 / 3}\right)$. The inflation theory thus becomes obsolete.

The problem of flatness $(\Omega=1)$ does not exist according to the $\mathrm{CTH}$, because the universe inevitably always leveled off 
into the unstable state of equilibrium of flatness (see "The labile equilibrium of cosmic expansion" section).

The CTH also has a plausible explanation for the problem of galaxy formation. Because of $\mathrm{G} \sim \mathrm{t}^{-2 / 3}$ and $\Delta \mathrm{\tau} / \Delta \mathrm{t} \sim \mathrm{t}^{-1 / 3}$ stars and galaxies formed much faster than according to established theories.

The problem of the world age dissolves in infinity, because according to the $\mathrm{CTH}$ there is no beginning of time. The "Big Bang" lies in the infinite past.

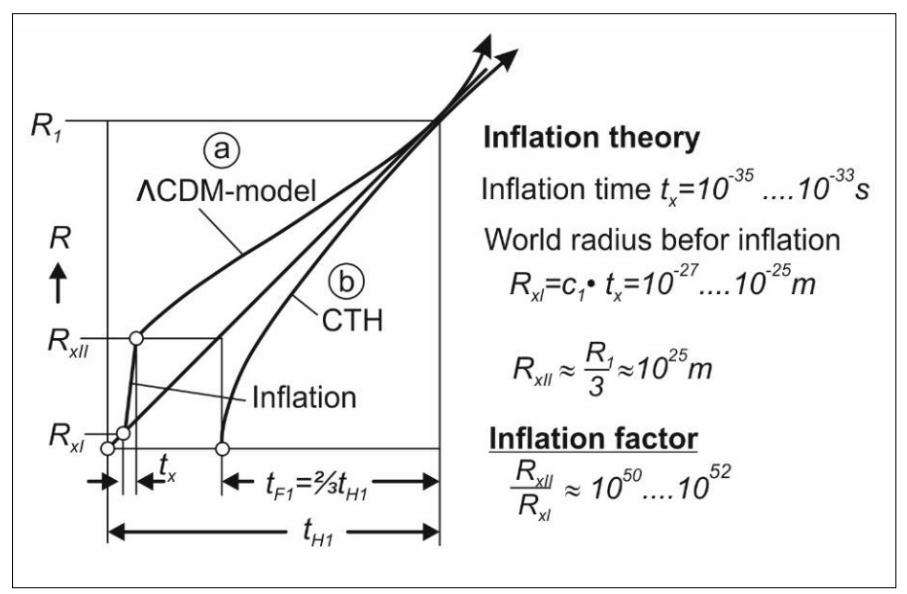

Figure 6. Expansion of the universe a) according to the Big Bang theory, b) after the CTH.

\section{Dark energy and the riddle of the cosmological constant}

There were two findings that led to the introduction of the "dark energy" and the reintroduction of the cosmological constant. Firstly, the measurement of the pattern of cosmic background radiation showed that the universe must be flat on a scale, which corresponds to an average density greater than the "dark" and making up visible matter together. Secondly, measurements on so-called type la supernovae (SNla) showed that the universe is expanding at an accelerated rate. The dark energy is supposed to be the missing energy necessary for a flat space - time $(\Omega=1)$ and it is supposed to generate an anti-gravitational force so that the universe can expanded faster and faster. The measurement result were interpreted as meaning that the SNla supernovae are further away from us than would be expected after the redshift of their light. This leads to the conclusion that the universe does not slow down as previously assumed, but accelerates. Since there is certainly no reason to doubt the measurement data - they have now been confirmed by many scientists - the question must be asked whether the data have been interpreted correctly so far. If one evaluates them according to the $\mathrm{TH}$, one surprisingly obtains completely different, much more plausible results. This will be briefly explained.

Hubble's law states that the escape velocity of a galaxy increases proportionally to its distance. The escape velocity of an object is determined by measuring the redshift $z$, the light emitted by it. According to the CTH, the speed of light was earlier (according to Newtons's time, not as a measured value) greater than today $\left(c=R \sim t^{-1 / 3}\right)$. Thus, with the same redshift, there are greater escape velocities $v$ and this greater distances than according to the conventional theory, and the further away the celestial body is, the greater the difference becomes. Figure 7 shows the measured data evaluated according to the $\mathrm{CTH}$ in comparison to conventional evaluations. An accelerated expansion of the universe is no longer recognizable from this, which also invalidates the main argument for the introduction of "dark energy". The SNla measurement results are therefore not a surprise, but a great success.

Also for explanation why universe is flat $(\Omega=1)$ dark energy is not needed. Because if one, as was explained in the "Cosmological Energy Balance" section, considers the space energy as positive energy, which is contained in the energy impulse tensor of Einstein's field equations, then the sum of all (positive) energies, despite $\Lambda=0$, is sufficient for a flat universe $(\Omega=1)$.

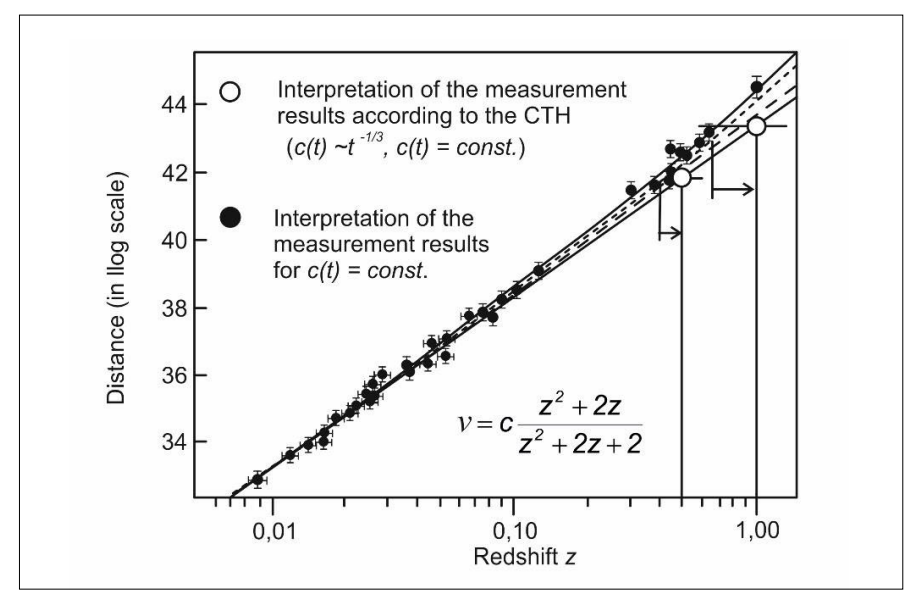

Figure 7. Hubble relationship according to measurement data from SNla [11], p. 18.

\section{The mystery of the cosmological constant}

"It is still unsolved today and perhaps the deepest unsolved fundamental problem of physics today" [10], S. 106.

Below we examine the consequences of setting $\Lambda=0$ in agreement with Einstein.

As already explained, the potential gravitational energy increases if the objects in the expanding universe are brought to greater distances from each other against the gravitational force. This requires energy, which is then stored in the newly created space as vacuum energy. Conversely, energy would be released if matter-filled spaces gravitatively implode or shrink. The space (the vacuum) thus contains positive energy! This is made available by the gravitational braking energy, which manifests itself in the decreasing expansion speed of the universe.

Starting from these considerations, the vacuum energy density can be calculated according to the $\mathrm{CTH}$ (see Appendix "Calculation of the vacuum energy density according to the CTH"). One receives:

$$
\begin{aligned}
& \varepsilon_{v}=3 / 4 \varepsilon \sim t^{-2} \\
& \text { ( } \varepsilon=\text { total energy density of the universe) }
\end{aligned}
$$

The vacuum energy (space energy) could also be understood as modern ether, similar to what Einstein 
formulated in 1920 at a lecture in Dutch Leiden. [3], p. 556 "The ether of general relativity is a medium which itself is barren of all mechanical and kinematic properties, but which co-determines the mechanical (and electromagnetic) evolution.

The mystery of the cosmological constant is that the value of the vacuum energy density calculated by quantum field theory (QFT) is about 120 powers of ten $\left(10^{120}\right)$ greater than it might be based on observations [13].

H. Goenner [14], p. 128, explains how vacuum energy density is calculated using QFT: "In the quantum field theories available, vacuum energy density generally diverges (ultraviolet divergence), i.e. the integral diverges over all wavenumbers $k$. This is the case with the QFT. To avoid infinitely large values, the $k$-space is cut off at an energy scale $E^{p} \approx 10^{19} \mathrm{GeV}$, i.e. at the Planck scale $\left(t_{p} \approx 10^{-43} \mathrm{~s}\right)$ ".

According to equation (14), the vacuum energy density at Planck's time $\left(\mathrm{t}_{\mathrm{p}} \approx 10^{-43} \mathrm{~s}\right)$ - the quantum field theory cuts off the diverging integral series at this point - results in a ratio value of

$$
\frac{\varepsilon_{v p}}{\varepsilon_{v 1}}=\left(\frac{t_{1}}{t_{p}}\right)^{2} \approx\left(\frac{10^{17}}{10^{-43}}\right)^{2}=10^{120}
$$

The exact numbers $\left(t_{1}=4.3 \cdot 10^{17} \mathrm{~s}, t_{p}=5.4 \cdot 10^{-44} \mathrm{~s}\right)$ result:

$$
\frac{\varepsilon_{v p}}{\varepsilon_{v 1}}=\left(\frac{4,3 \cdot 10^{17}}{5,4 \cdot 10^{-44}}\right)^{2}=0,64 \cdot 10^{122}
$$

This is an amazing result and it solves one of the biggest problems of modern physics!

In summary, the CTH demands a completely new interpretation of the term "vacuum energy":

The vacuum energy density is positive and timedependent $\left(\varepsilon_{v} \sim t^{-2}\right)$, not negative and constant in what today's doctrine is based.

The cosmological constant does not exist in reality $(\Lambda=0)$, therefore there are also no "dark energy" $\left(\Omega_{\Lambda}=0\right)$.

The vacuum energy is a component of the total energy of the universe and in the energy impulse tensor (Tik) of Einstein's field equations. $\Lambda=0$ means: The $C T H$ gets along with one free parameter less than the $\Lambda C D M$ model!

\section{Expansion of local, gravitatively bound structures}

The orbits of the planets orbiting around the sun are ellipses, which are in a stable state of equilibrium, i.e. centrifugal force and gravitational force balance each other out. However, this state is not stable in the long term. Since the gravitational constant after the CTH decreases with time $\left(G \sim t^{-2 / 3}\right)$, the orbit radius $r$ will slowly increase over time and expand according to the same law as the universe (Figure 8).

$$
r \sim R \sim t^{2 / 3}
$$

This result does not violate the current state of knowledge [15], p. 56: "In fact, the experts still disagree today as to whether the space within galaxies or even the space between the planets of our solar system does not expand as well". For example, it has been observed that small gravitatively bound galaxy clusters do not continue to compress, as would be expected, but the exact opposite happens. They move away from each other, [16], p. 114: "It is almost absurd that Hubble's law is observed in very small groups of galaxies as well (as on very large distances), even with the same value $H_{0}$ ".

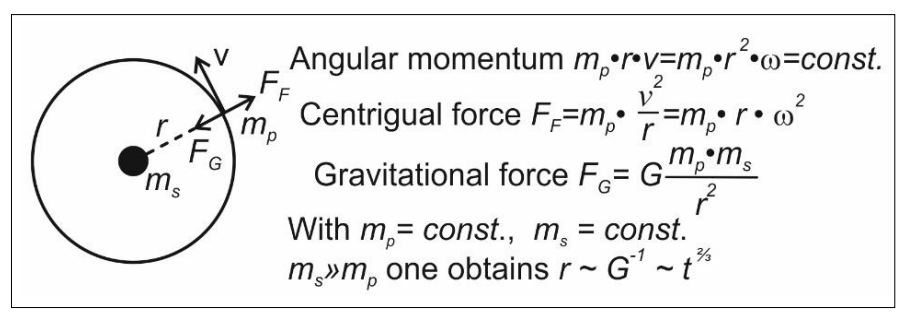

Figure 8. Expanding local structures.

So you have to assume from this that such galaxy groups first formed in a stable state of motion, which then slowly expands again according to $r \sim t^{2 / 3}\left(G \sim t^{-2 / 3}\right)$. Thus also the moon should move away from the earth. According to the CTH this would be about $2.7 \mathrm{~cm} /$ year. Measured were $3,8 \mathrm{~cm} /$ year. The difference might be to be led back on brake forces by the tides.

\section{Is there "Dark Matter"?}

As the experimental results show, there seems to be "dark matter" or, alternatively, a slightly stronger gravitational force only at a greater distance from us as a measurable quantity.

Since we always look back into the past when looking at galactic objects, we see them in the state in which they were as many years ago as they are light years away from us.

According to the $\mathrm{CTH}$, gravity used to be stronger than it is today $\left(G \sim t^{-2 / 3}\right)$. Near objects should therefore - as also measurements prove - hardly provide an indication of "dark matter". However, the greater the distance, the greater the observed deviation from Newton's law of gravity ( $\mathrm{G}=$ constant). The most important measuring method to discover "dark matter" is the gravitational lens effect. It measures the deflection of light caused by massive objects located between earth and distant galaxies or groups of galaxies. A deviation from $\mathrm{G}=$ constant should be particularly noticeable. It would therefore be necessary to check whether a decreasing gravitational constant $\left(G \sim t^{2 / 3}\right)$ could explain the experimental results. If this were possible, then there would be no dark matter at all.

\section{The hypothesis of the "Large Numbers"}

Paul Dirac noticed that there are two extremely large, almost identical numbers, one of which comes from the subatomic world and expresses the ratio of electromagnetic force $\left(F_{e}\right)$ and gravitational force $\left(F_{G}\right)$ between nucleons and electrons $\left(N_{1}=F_{e} / F_{G}=e^{2} / G \cdot m_{n} \cdot m_{e} \approx 10^{39}\right)$ and the other measures the scale of the universe $\left(\mathrm{N}_{2}=\mathrm{R} / \mathrm{r}_{\mathrm{e}} \approx 10^{41}\right)$ [17], $\mathrm{p}$. $521 \mathrm{ff}$ ( $\mathrm{e}=$ charge of the electron, $m_{n}=$ mass of the nucleon, $m_{e}=$ mass of the electron, $r_{e}=$ electron radius, $G=$ gravitational constant, $R=$ cosmic radius).

According to current theories, $N_{1}$ should be constant, but $N_{2}$ should grow with the expansion of the universe.

Dirac did not believe that equality $\left(N_{1} \approx N_{2}\right)$ is only a 
coincidence of the moment. In order for $N_{1} \approx N_{2}$ to be valid at all times, he suggested that the gravitational constant should decrease as the size of the universe increases ( $G \cdot R=$ constant).

Since there are strong arguments against Dirac's hypothesis [17], p. 532/533, it has not yet been accepted. The $\mathrm{CTH}$ offers an alternative to the Dirac hypothesis. Dirac's hypothesis is based on the electromagnetic force $F_{e^{\prime}}$ which has a range of the size of the universe $R$. The electromagnetic force $F_{e}$ is the largest of the two forces. The question is whether it would not be more sensible to choose a reference force whose range is identical to the electron radius. Exactly that would apply to the strong nuclear force $F$ s. It has a range of about $10^{-15} \mathrm{~m}$ (Yukawa radius), which also corresponds to the size of an elementary particle.

Since the strong nuclear force $F s$ is greater by a factor of $10^{41}$ than the gravitational force $F_{G}[18]$, p. 971 , there is an almost exact correspondence between $N_{1}$ and $N_{2}$.

$$
N=N_{l}=N_{2}=F_{s} / F_{G}=R / r_{e}=\left(t / t_{p}\right)^{2 / 3}
$$

Today ( $t=t_{1}=13.7 \cdot 10^{9}$ years) $\mathrm{N}$ has the numerical value $\approx 10^{41}$.

It is obvious to establish a relationship between gravitational force and strong nuclear force because there is a special relationship between the two forces. B. Greene points out that [6], p. 155: " ... the gravitational force and the strong force have very different properties, but that they have a similar function: Both are necessary for the universe to have certain symmetries. The same is true for the weak and electromagnetic force: their existence is also bound to certain symmetries of calibration".

One should also consider that the accuracy with which $G$ could previously be measured is only $1.5 \cdot 10^{-3}$ [19]. This is many powers of ten less accurate than the numerical value of other natural constants.

According to the $\mathrm{CTH}\left(G \sim t^{-2 / 3}\right)$ the relative decrease of $G$ today is about $7 \cdot 10^{-11}$ per year! The lunar laser rating experiment recently showed that the value of $G$ would have to change by less than $10^{-6}$ per year in order to no longer be measurable. That would be more than 10,000 times the annual change resulting from the CTH! A direct experimental proof, whether $G$ is constant or variable, is still a long way off.

\section{Unification of gravity with the strong nuclear force}

The theory of supersymmetry (supergravity) postulates that shortly after the "Big Bang" (until Planck's time) all four basic forces of nature (strong and weak nuclear force, electromagnetic force, gravitational force) were united in a single primary superpower (Figure 9).

At Planck's time $\left(t_{p} \approx 10^{-43} \mathrm{~s}\right)$, according to theory, the first symmetry calculation took place, with gravity "crystallizing" as the first force.

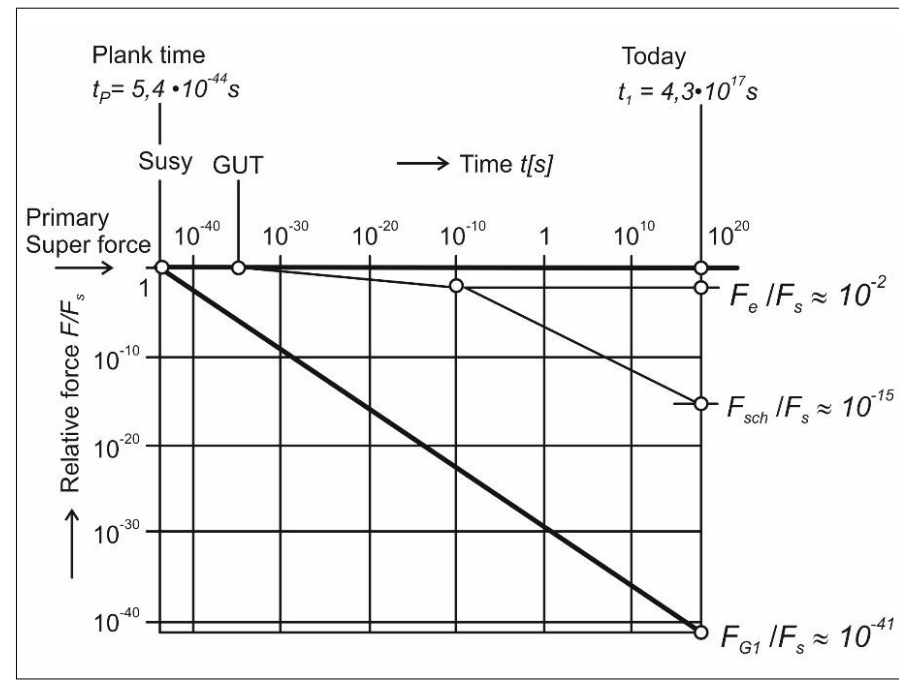

Figure 9. The fundamental forces of nature: $F_{s}$ : strong nuclear power, $F_{\text {sch }}$ : weak nuclear power, $F_{e}$ : electromagnetic force, $F_{G}$ : gravitational force.

Through later symmetry breaks, the other forces of nature also became independent. Today the strong nuclear force, as already mentioned, is $10^{41}$ times stronger than the gravitational force. Since the latter changes proportionally to the gravitational constant, according to the $\mathrm{CTH}$ it must have been greater at Planck's time by the factor $\left(t_{1} / t_{p}\right)^{2 / 3}=\left(4,3 \cdot 10^{17} /\right.$ $\left.5,4 \cdot 10^{-44}\right)^{2 / 3} \approx 10^{41}$ than today, i.e. it was then identical with the strong nuclear force, as required by the theory of supergravity. This surprising result gains even more fascination if one links the strength of the two forces to their respective ranges. From eq. 18 follows:

$$
F_{S} \cdot r_{e}=F_{G} \cdot R
$$

Thus the universe at Planck's time had the size of an elementary particle $\left(R_{p}=r_{e} \approx 10^{-15} \mathrm{~m}\right)$, which by the way corresponds to the Planck length $I_{p}$ at Planck's time $t_{p}$ according to the $\mathrm{CTH}$, as shown below.

The Planck length $I_{p}$ is defined as the length that the light travels in the Planck time $t_{p} \approx 10^{-43} \mathrm{~s}$. The Planck length $I_{p}$ is defined as the length that the light travels in the Planck time $t_{p} \approx 10^{-43} \mathrm{~s}$. The Planck length $I_{p}$ is defined as the length that the light travels in the Planck time $t_{p} \approx 10^{-43} \mathrm{~s}$. It is today $\left(t=t_{1}\right): I_{p 1}=c_{1} t_{p}=3 \cdot 10^{8} \cdot 10^{-43} \approx 10^{-35} \mathrm{~m}$.

According to the $\mathrm{CTH}, c \sim t^{-1 / 3}$ applies. The speed of light at Planck's time was thereafter by the factor $\left(t_{p 1} / t_{p}\right)^{1 / 3} \approx$ $\left(10^{17} / 10^{-43}\right)^{1 / 3} \approx 10^{20}$ larger than today $\left(t_{1} \approx 10^{17} \mathrm{~s}\right)$.

You get for the Planck length at Planck time: $I_{p 1}=c_{1} t_{p}=$ $3 \cdot 10^{28} \cdot 10^{-43} \approx 10^{-15} \mathrm{~m}$.

It would thus be identical with the size of the universe at Planck's time and the "elementary length" of particle physics (Yukawa radius, Compton wavelength of the proton, classical electron radius).

It is also interesting with regard to the relationship between gravitation and strong nuclear force that, according to loop quantum cosmology, the gravitational force at the 
Planck scale $\left(I_{p} \approx 10^{-15} \mathrm{~m}\right)$ is said to have had a repulsive effect [20], p. 155, which also applies to strong nuclear force (Figure 10).

This similarity between gravitational force and strong nuclear force suggests that before the first symmetry calculation $\left(t<10^{-43} \mathrm{~s}\right)$ there was indeed a repulsive super force that could have triggered the expansion of the universe. A quote from B. Greene [6], [p. 311/312]: "The surprising discovery was made that gravitation can have a repulsive effect under certain conditions, and according to the theory exactly these conditions prevailed in the earliest moments of cosmic history. During a time interval when a nanosecond would appear to be eternity, the universe offered conditions in which gravity could exert its repulsive effect so violently that each region of space was driven away by each other with tremendous force. The repulsion of gravity was so violent that not only was the bang identified, but it also proved to be larger - much larger - than anyone had dreamed".

Such extreme events could possibly also be explained by the $\mathrm{CTH}$. It requires that the expansion velocity of the universe $(\dot{R}=c)$ at Planck's time be greater by a huge factor of $10^{20}$ than it is today. In order to generate this enormous expansion velocity, the primordial, repulsive super force would indeed have had to have been unimaginably large, which seems quite plausible if one assumes that it had a similar course to the strong nuclear force at that time (Figure 10). Relative to the cosmic (real) time $\tau$, this expansion process took place much more slowly than it did today.

The close relationship between strong nuclear force and gravitational force could perhaps be a starting point for finding a formulation of quantum gravity that describes the universe within the framework of quantum cosmology. This would be a new, hitherto untested step towards the unification of quantum physics and GTR.

The theory of the Standard Model of elementary particles covers the world of subatomic particles [21], p. 68: "It contains all fundamental forces - with the exception of gravity, which does not seem to fit into the framework".

But since - as has been shown - the CTH links the strong nuclear force $\left(F_{S}\right)$ with the gravitational force $\left(F_{G}\right)\left(F_{G}=F_{S} \cdot r e\right.$ $\left.\cdot R^{-1} \sim t^{-2 / 3}\right)$, gravity could perhaps find its place within the framework of the Standard Model. However, then a timedependent quantity $\left(F_{G} \sim t^{-2 / 3}\right)$ with three constant quantities (electromagnetic force, strong and weak nuclear force) would have to be brought into a consistent relationship with each other. At present, it is not foreseeable how this will be achieved.

But if it were possible, time - in contrast to today's view - would play just as important a role in elementary particle physics as it does in cosmology.

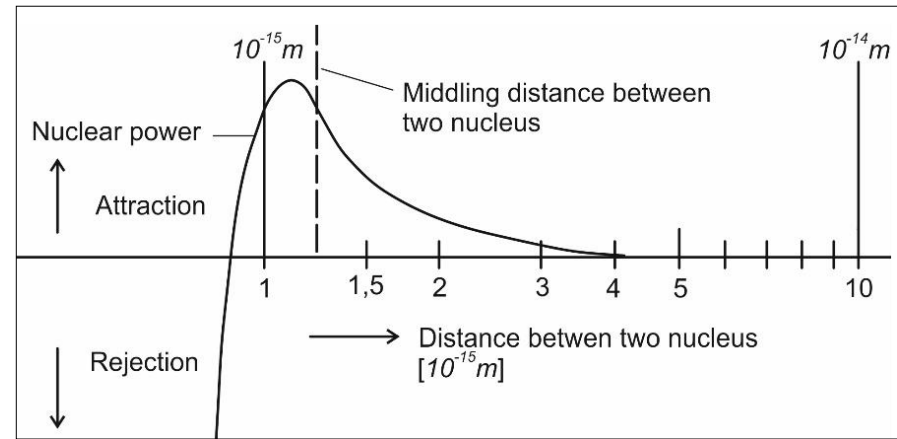

Figure 10. Strength of nuclear forces depending on Removal of two Nucleons of another [21], p. 674.

\section{Reflections on the first and second Machian principles}

The term "Machian Principle" is associated with a wide variety of contents and there is currently no uniform wording for it [17]. p. 507: "Nobody is quite sure what Mach's principle actually means, and everyone has a different interpretation. Every change in the value of $G$, no matter how small, can be seen as proof of a Mach effect. Skalar tensor theory allows the $G$ variation to be either large or small, and if we adjust the $G$ button so that the $G$ variation is small enough never to conflict with observation, then we can say that the universe follows Mach's principle".

The question now is whether and in what way the "first Machian principle" is compatible with the CTH. One statement of this principle is [17], p. 281: "... that all inertial forces are determined by the total amount of matter in the universe and are proportional to it".

How the influence of all masses of the world on the inertial motion of every single body comes about and which laws it satisfies, leaves Mach to future experience. Occasionally, however, he speaks of an influence independent of distance, so that the (few) near masses have only little effect compared to the (many) distant ones. In fact, it can be proven that in a continuum the gravitational forces between a single mass and the masses distributed in the continuum are independent of distance (Figure 11).

The universally acting gravitational forces must remain constant according to both the $\mathrm{CTH}\left(M \sim R, G \sim R^{-1}, G M=\right.$ const. $)$ and conventional theories ( $M=$ const., $G=$ const.). This is also in line with the results of the Viking space probes, which (among others) should refute or confirm the Dirac hypothesis (timevarying "gravitational constant") [22], p. 539/540: "Until today we have no evidence that gravity becomes weaker in the course of time. The Viking missions to Mars have shown that gravity, if it changes over history, may have fluctuated by no more than $1 \%$ throughout the 15 billion year history of the universe. That is one hundredth of the value predicted by Dirac".

Since in such experiments only the product of gravitational constant $(G)$ and mass $(M)$ can be measured [23], the following conclusions can be drawn:

a. If the established theories are valid, the Viking measurements confirm neither the Dirac hypothesis nor the Mach principle nor the Scalar tensor theory. 
b. If the CTH is valid, the measurement results are compatible both with the Scalar-Tensor-Theory and with Mach's principle.

The CTH can thus be brought into agreement with the first Machian principle or the scalar tensor theory. E.R. Harrison also pleads for not abandoning scalar tensor theory without good reason [17], p. 508: "The advantage of scalar tensor theory is that it forces us to make careful observations and experiments in the hope that one day we will know how to interpret the Einstein equation".

From this point of view, the CTH would be an extended interpretation of Einstein's field equations, including the 1st Machian principle.

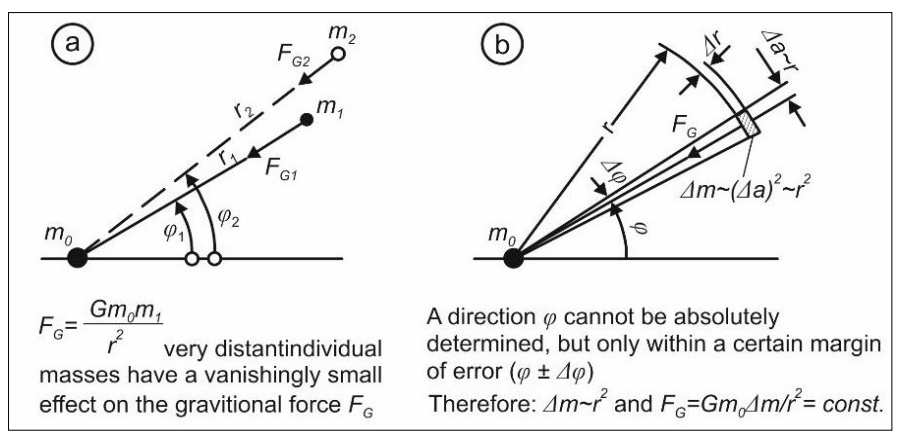

Figure 11. Dependence of gravitational force from a distance a) between two individual masses (b) between a single mass and a mass continuum

The "second Machian principle" is derived from the assumption expressed by Ernst Mach [24], p. 25: "... that the "entropy of the universe" could serve as a measure for absolute time". In order to concretize this statement, however, [24], p. 25: "first of all it should be clarified that certain cosmic state changes (of entropy, of the world radius) have a causal effect on the course of local state changes.

Furthermore, it should be clarified which of the known interactions bring about this influence. Only through these additional clarifications could the "second Machian principle" perhaps take on a form in which it can be confronted with the material of experience and with the existing theories".

It also remains unclear [7]: "whether and in which way the entropy of the world can influence the time direction and the course of all concrete clocks, which make it possible to measure this absolute time more or less well".

\section{Relationship between cosmic time $(\tau)$, cosmic entropy (SK) and cosmic radius (R)}

Like the CTH, llya Prigorgine assumes a continuous process of producing matter. From this he concludes [7], p.289: "an entropy generation proportional to the rate of pGTRicle generation" $\left(d S_{K} / d t \sim d M / d t\right)$.

From the relations (6), (7) and (10) one obtains

$$
\frac{d M}{d t} \sim \frac{d R}{d t} \sim \frac{d \tau}{d t} \sim \frac{d S_{K}}{d t} \sim t^{-1 / 3}
$$

This means, in tune with Prigorgine, an initial explosive entropy increase [7], p.297.

As a measure for the cosmic time ( $\tau)$ the cosmic entropy $\left(S_{K}\right)$ or the cosmic radius $(R)$ can actually serve, as Ernst Mach assumed, if one takes the statements of Prigorgine seriously. Also the time direction (time arrow) is fixed with it, because both $S_{K}$ and $R$ can become bigger with the time only, never smaller. The thermodynamic and cosmic time arrows are therefore identical.

According to P. Mittelstaedt the Machian assumption should be understood [24], p. 25: "... that cosmic entropy has a causal effect on the course of all other clocks in an unknown way".

Actually it can be proved that real clocks (e.g. pendulum clocks and atomic clocks) do not measure Newton's time $t_{\text {, }}$ but exactly the cosmic time $\tau$, if they "tick" according to the laws of the CTH (see "Solving old big bang theory problems (horizon, flatness, galaxy formation and age problem" section).

\section{Epilog}

To this day, we have no clear idea what time means in the physical sense. Many scientists believe (like Einstein) that time is an illusion. Purely subjectively, however, we feel that there should be a stream of time that irreversibly flushes us from the past into the future. So what is time? We are still trying to find a satisfactory answer to this ancient human question.

Roger Penrose once commented on the current time problem [25]: "In my opinion, our current picture of physical reality, especially with regard to the nature of time, is responsible for a great deal of confusion, perhaps even greater than that caused by today's theory of relativity and quantum mechanics".

The disappearance of time in modern physics (block universe) is based on the fact that the laws of mechanics, relativity and quantum mechanics do not have a given time direction (=time arrow). Any process obeying these laws could also be reversed, i.e. it would be time-reversible.

In contrast, the asymmetric cosmic time has a given direction, thus it is not reversible and therefore not eliminable. Such a cosmic time would have the consequence that the laws of nature are not strictly determined. This would be necessary in order to explain the various evolutionary development processes that we observe everywhere. They are based on unpredictable spontaneous mutations, which are limited by the selection pressure of the environment to an extent necessary for evolution. There are also fluctuations on a cosmic scale, e.g. deviations from the theoretically required expansion speed of the universe. However, they are kept within limits because (according to the $\mathrm{CTH}$ ) the universe always leveled off into the unstable state of equilibrium that we observe today (see "The labile equilibrium of cosmic expansion" section). Such a time-afflicted "dynamic instability" [26] is characteristic for many natural events. Therefore it is difficult for us to accept a timeless universe. Prigogine also does not believe in a timeless world [7], p. 310: "Time cannot spring from timelessness. The timeless laws of physics cannot be accepted as a true "reflection" of the fundamental truth of the physical world, because this truth makes us strangers in this world and reduces the diversity of the phenomena we observe to a mere appearance". 
Stephen Hawking solved the time problem pragmatically. According to his conception [9], p. 177: "... a scientific theory is nothing more than a mathematical model that we design to describe our observations: It exists only in our heads. Therefore it is pointless to ask: What is real, the "real" or the "imaginary" time? It's just which of them is the more useful description". This is exactly how the time of the CTH is to be understood, i.e. it defines time according to the criterion of usefulness, but without being able to grasp its essence. In the past, there have been several attempts to define the concept of time in such a way that nature can be described as simply and comprehensively as possible. Poincarè, for example, demanded a measure of time that also satisfies aesthetic requirements [24], p. 27: "Time must be defined in such a way that the equations of mechanics become as simple as possible".

Peter Mittelstaedt later combined the principle of simplicity with another demand, namely [24], p. 32: "... that the time parameter should be chosen in such a way that the explanatory power of the theory becomes as large as possible" The "asymmetric cosmic time" fulfils both requirements!

As already explained before, the CTH is not only included in the GTR, but is even demanded by it, if it claims validity for the whole universe. However, then the laws of nature must change in the course of time, as prescribed by the CTH.

Lee Smolin is also convinced that there are no iron laws of nature [8], p. 15: "Laws are not timeless. Like everything else, they are properties of the present and can evolve over time". By the way, he points out that this thought is not new. Already Paul Dirac conjectured [8], p. 28: "At the beginning of time the laws of nature were probably very different from what they are now. We should therefore bear in mind that the laws of nature change continuously over time, rather than being uniform throughout space-time".

Although the $\mathrm{CTH}$, which was developed more than 20 years ago, offers surprising solutions to many cosmological problems, such as solving the mystery of the cosmological constant, which is thought to be perhaps the deepest unresolved fundamental problem in physics today [10], $\mathrm{p}$. 106 , it has hardly been acknowledged so far. This may be due to the fact that her statements stand in stark contrast to the mainstream and also to the fact that she disregards supposedly "secured knowledge" too disrespectfully.

The scientific progress that results from the CTH lies in the extension of the concept of time, whereby many natural phenomena that cannot be explained with today's understanding of time can be explained. (See also: The most important results of the $\mathrm{KHZ}$, page 1 ).

In view of the difficulties that cosmology is facing today, it would be urgently necessary to finally start a discussion that also includes alternatives to the current big bang theory. Because [27]: "More and more questions are piling up, which almost already shake the foundations of cosmology". Some scientists therefore suspect [28]: "Perhaps the solution to our questions, our riddles, is also one that cannot be clarified by an experiment by physicists or an observation by astronomers, but by a completely new approach, by another theory". Hermann
Nicolai pointed out this problem many years ago [29]: "We have a wealth of data on the nature of the universe. But a comprehensive theory that could explain all these observations is not in sight at present".

The CTH could be an important step towards such a fundamental theory.

As is well known, our physical theories describe the world as we can grasp it through our conceptual ideas (hypotheses) and sensory perceptions (experiments). Or (according to Wittgenstein): Our theories depict reality as it appears to the gaze from the closed window of our inner world. Whether reality - the thing itself, as Kant put it - is actually as it can be fathomed by man with his limited abilities is therefore questionable. Nevertheless, we must believe that new scientific hypotheses will bring us closer to the "truth" again and again, because otherwise all science would be meaningless.

\section{Finally, a few summarising remarks: \\ Definition of physical quantities}

In order to be able to define physical quantities clearly, they must be related to unambiguous terms. Example: The relative times of SRT and GRT are related to abstract Newtonian time (here and now) to make them understandable. This also applies to cosmic time $\tau\left(\tau \sim \mathrm{t}^{2 / 3}\right.$, $\Delta \tau / \Delta t \sim t^{-1 / 3}$ ).

\section{Cosmological constant $\Lambda$}

From the solutions of the original Einstein field equations one obtains an expanding universe (Friedmann). But Einstein, like almost all of his contemporaries, believed that the universe was static and inserts the cosmological constant $\Lambda$ (=integration constant) into his field equations to stabilize it. When proof was furnished that the universe was actually expanding (Hubble), Einstein eliminated the cosmological constant again from the field equations [1], p. $110 \mathrm{ff}$. and described its introduction as the greatest folly of his life. Later, when the results of measurements on SNla supernovae showed that the universe is supposedly expanding at an accelerated rate today, the cosmological constant was reintroduced $(\Lambda>0)$. The $\mathrm{KZH}$ now eliminates $\Lambda$ for the second time $(\Lambda=0)$, with which Einstein would surely have agreed.

\section{Time: Illusion or Reality}

Einstein interpreted his field equations from the point of view of time symmetry, which is also the basis of Newton's theory of gravity. From this he concluded that time is an illusion. This is also expressed in the current "block universe", in which the history of the universe is considered as a whole. In fact, however, the field equations of the GRT - as shown by the $\mathrm{CTH}$ - require a time asymmetry. Such an asymmetric cosmic time has - just like time in thermodynamics - a direction of flow (time arrow). The laws of nature are therefore not timeless! They obey the law of universal entropy increase.

Question: Are Einstein's equations smarter than Einstein was? 


\section{Mathematical Appendix}

\section{Rate speed of watches according to CTH}

Pendulum clocks: For the period $\mathrm{T}$ of oscillation of a pendulum in the gravitational field of the earth (or in any other gravitational field) applies.

$$
T=2 \pi \sqrt{\frac{l}{g}} \sim g^{-1 / 2}
$$

( $I=$ pendulum length, $g=$ acceleration due to gravity)

With $g \sim G$ and $G \sim t^{-2 / 3}$ you get $T \sim t^{1 / 3}$, which corresponds to a clock frequency of $f=1 / T \sim t^{-1 / 3}$

Atomic clocks: For the inneratomar oscillation frequency $f$ of an atomic clock, [23] $f=c / \lambda$ the wavelength $\lambda$ is a characteristic quantity associated with the atomic species. The numerical values for caesium atomic clocks are [23]: $\lambda \approx$ $3 \mathrm{~cm}, f \approx 10^{10} \mathrm{~s}^{-1}$ (exactly $9192631770 \mathrm{~s}^{-1}$ ). If the speed of light would change according to the CTH $\left(c \sim t^{-1 / 3}\right)$, the oscillation frequency of the atomic clock would also change linearly, i.e., $f \sim t^{-1 / 3}$.

So pendulum clocks and atomic clocks, if they "tick" according to the laws of the $\mathrm{CTH}$, do not measure Newtonian time, but cosmic time.

\section{Calculation of the vacuum energy density according to the CTH}

From the law of conservation of energy

$E=M(t) c^{2}(t)=$ const.

is obtained by differentiating

$d E / d t=\dot{E}=\dot{M} c^{2}+2 M c c=0$

As already mentioned, the material energy $M$ contains all gravitatively effective forms of energy, including vacuum energy.

Which part of the total energy $M$ is contained in the vacuum can be determined by a simple observation. The time gradient $E_{v}$ for the gravitational energy can be determined with the aid of an idealized model of the universe, in which all forms of energy are homogeneously distributed and expand at a speed proportional to the distance (Figure 12). The energy which is released in the radial direction (= one-dimensional) by the delayed expansion of the matter $\mathrm{dm}$ contained in a spherical shell with radius $r$ and thickness $d s$ and which transforms into space energy, is

$$
d\left(d E_{r v}\right)=d F \cdot d s
$$

Since the room enlargement is not only radial (onedimensional), but three-dimensional, the following applies:

$$
d\left(d E_{V}\right)=3 \cdot d\left(d E_{r V}\right)=3 \cdot d F \cdot d s
$$

This braking energy is converted into room energy (vacuum energy) as the room expands.

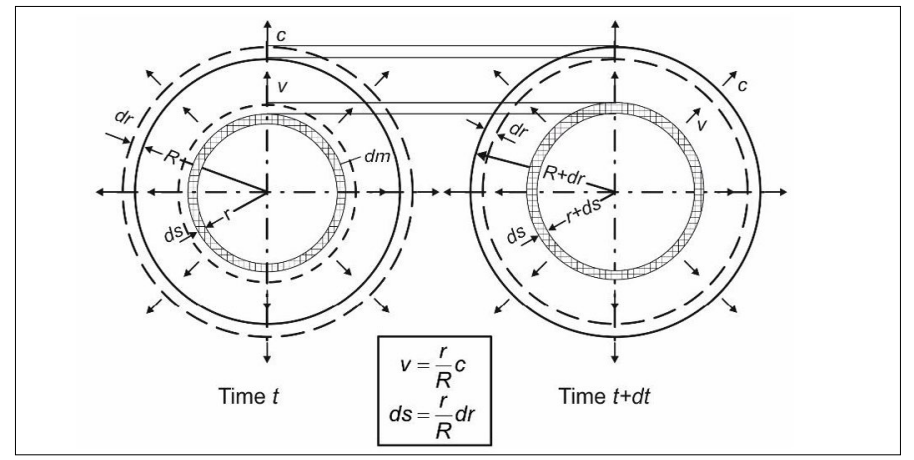

Figure 12. Temporal Change in space energy.

$$
\begin{aligned}
& \text { With } \quad d F=-d m \dot{v}=-d m \frac{r}{R} \dot{c}, \quad d s=\frac{r}{R} d r=\frac{r}{R} c d t \\
& \text { and } \quad d m=4 r^{2} \pi \rho d s=4 r^{2} \pi \rho \frac{r}{R} d r \\
& \text { results in } d \dot{E}_{v}=\frac{d\left(d E_{v}\right)}{d t}=-12 \pi \rho c \dot{c} \frac{r^{5}}{R^{3}} d r
\end{aligned}
$$

By integrating $r=0$ to $r=R$ one obtains

$$
\begin{aligned}
& \dot{E}_{Y}=-\left.\frac{12}{6} \pi c \dot{c} \rho \frac{r^{6}}{R^{3}}\right|_{r=0} ^{r=R}=-2 \pi \rho c c R^{3} \\
& \text { Inserting } M=\frac{4}{3} \pi \rho R^{3} \text { leads to } \\
& \dot{E}_{Y}=-\frac{3}{2} M c \dot{c}
\end{aligned}
$$

From the comparison of equation (23) and (26) it follows that the energy released by the delayed expansion converts to $1 / 4$ into normal matter and to $3 / 4$ into space energy. When the universe began to exist in the "Big Bang", the concrete forms of energy $M=E / c^{2}$ were small. The ratio of normal matter to space energy must therefore always have been 1:3 for each time thereafter. For the vacuum energy density follows from it:

$$
\varepsilon_{v}=3 / 4 \varepsilon \sim t^{-2}
$$

The vacuum energy density is thus a component of the (positive) total energy in the universe, of which the share of $\varepsilon \mathrm{V}$ corresponds approximately to that which also applies to the $\Lambda C D M$ model $(\Omega M \approx 0.25, \Omega V \approx 0.75)$.

\section{Kinetic and potential energy of planets [12], p. 55}

Assertion: The kinetic energy that a planet possesses on its orbit around its central mass (star) is approximately the same as the energy that would be used to remove it from the gravitational field of the central mass.

Proof: The kinetic energy of a planet orbiting a star is (Figure 13)

$$
E_{\text {kin }}=m_{p} \cdot \frac{v_{p}^{2}}{2}
$$

The potential energy that a planet has at a distance $(r)$ from its orbit radius $r_{p}$ is

$$
E_{p o t}=G m_{s} m_{p} \int_{r_{p}}^{r} \frac{d r}{r^{2}}=-G m_{s} m_{p}\left(\frac{1}{r}-\frac{1}{r_{p}}\right)
$$

for the limiting case $r=\infty$ : $E_{p o t}=G m_{s} m_{p} / r_{p}$ 


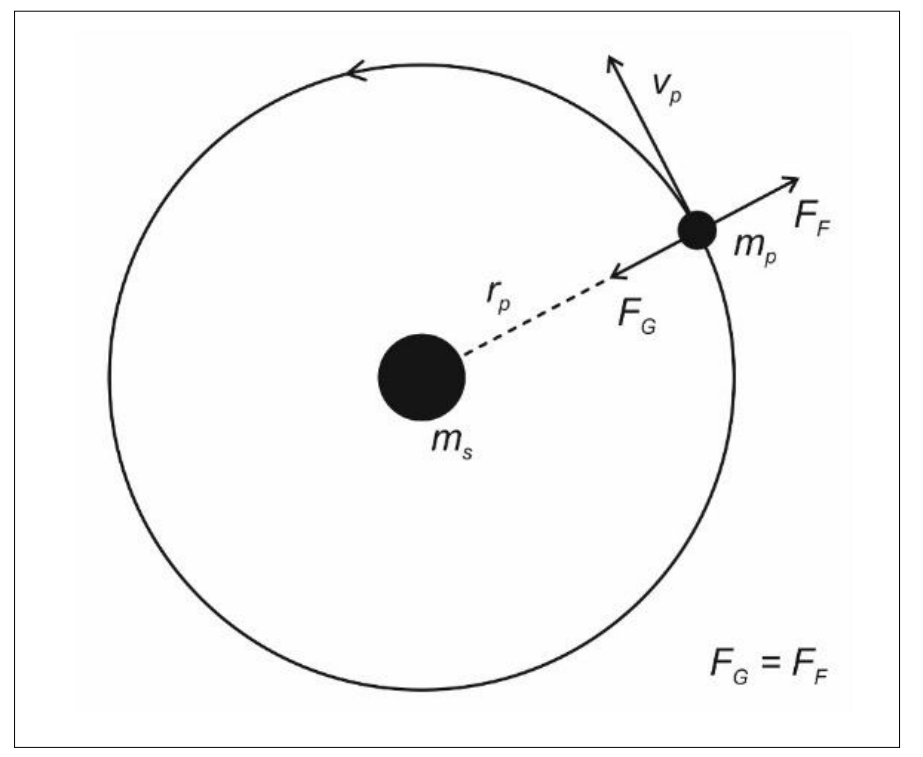

Figure 13. Planet $\left(m_{p}\right)$, which is circles a star $\left(m_{s}\right)$. Example 1: earth - Sun

Example 1: earth - sun

$$
\begin{aligned}
& G=6,7 \cdot 10^{-11} \frac{\mathrm{m}^{3}}{\mathrm{kgs}^{2}}, m_{p}=6 \cdot 10^{24} \mathrm{~kg}, m_{s}=2 \cdot 10^{30} \mathrm{~kg}, \\
& r_{p}=1,5 \cdot 10^{11} \mathrm{~m} \\
& v_{p}=\frac{2 \pi r_{p}}{t}, t=1 \mathrm{Jahr}=3 \cdot 10^{7} \mathrm{~s}, v_{p}=\frac{2 \pi \cdot 1,5 \cdot 10^{11}}{3 \cdot 10^{7}} \approx 3 \cdot 10^{4} \mathrm{~ms}^{-1} \\
& E_{\text {kin }}=\frac{6 \cdot 10^{24} \cdot\left(3 \cdot 10^{4}\right)^{2}}{2} \approx 3 \cdot 10^{33} \frac{\mathrm{kgm}^{2}}{\mathrm{~s}^{2}}
\end{aligned}
$$

\section{Example 2: Moon - earth}

$$
\begin{aligned}
& M_{p}=7,35 \cdot 10^{22} \mathrm{~kg}, \quad r_{p}=3,84 \cdot 10^{8} \mathrm{~m}, \quad t \approx 1 \text { Monat }=2,36 \cdot 10^{6} \mathrm{~s} \\
& V_{p}=\frac{2 \pi \cdot 3,84 \cdot 10^{8}}{2,36 \cdot 10^{6}} \approx 10^{3} \mathrm{~m} / \mathrm{s} \\
& E_{\text {kin }}=\frac{7,35 \cdot 10^{22} \cdot 10^{6}}{2} \approx 3,7 \cdot 10^{28} \frac{\mathrm{kgm}^{2}}{\mathrm{~s}^{2}} \\
& E_{\text {pot }}=\frac{6,7 \cdot 10^{-11} \cdot 6 \cdot 10^{24} \cdot 7,35 \cdot 10^{22}}{2,36 \cdot 10^{6}} \approx 7,7 \cdot 10^{28} \frac{\mathrm{kgm}^{2}}{\mathrm{~s}^{2}}
\end{aligned}
$$

Note: $E_{\text {kin }}$ is a bit smaller than $E_{\text {pot }}$ because in the formation of the systems Sun - Earth and Earth - Moon the satellites were not captured from an infinite distance from the central body and because during and after the formation process collisions with other celestial bodies (asteroids) took place which could have reduced $E_{k i n}$.

\section{Conclusion}

As is well known, Einstein put time into perspective twice. In special relativity (the dependence of time on relative velocity) and in general relativity (all bodies fall in the direction in which the time slows down).

The Cosmic Time Hypothesis $(\mathrm{CTH})$ requires that time must be relativized a third time in order for the GR (General Theory of Relativity) to be valid for all events in the universe. According to the $\mathrm{CTH}$, the clock frequency of time was higher than it is today and will slow down in the future. This asymmetry gives time a direction (cosmic time arrow), which can be used to explain many evolutionary processes in the cosmos that the current standard model of cosmology ( $\triangle C D M$ model) cannot explain. The $\mathrm{CTH}$ thus has far-reaching consequences for our entire physical worldview.

\section{Formula Symbols}

c: velocity of light in the vacuum; $c_{S}$ : velocity of sound; $E$ : total energy of the universe; $f$ : cycle frequency of time, energydegree of freedom; F: force; G: gravitational constance; h: Hubble-Expansion ( $=\mathrm{H} / \mathrm{C}=1 / \mathrm{R})$; $\mathrm{H}$ : today's Hubble-constancy $\left(=1 / t_{H}=c / R\right) ; m$ : mass; $M$ : total mass oft he university $\left(=E / c^{2}\right)$; $\mathrm{p}$ : pressure; q: deceleration parameter; $\mathrm{r}$ : radius; $\mathrm{R}$ : world radius; $\mathrm{s}$ : way; $\mathrm{t}$ : "now-time" = Newtonian time; $\mathrm{t}_{\mathrm{H}}$ : Hubbletime $(=\mathrm{R} / \mathrm{c})$; T: temperature; v: relative velocity; $\mathrm{V}$ : volume; $\mathrm{x}$ : spatial coordinate; $\varepsilon$ : total energy density oft the universe; $\varepsilon_{\mathrm{v}}$ : vacuum energy density; $\mathrm{\kappa}$ :coupling constant $\left(=8 \pi G / c^{2}\right) ; \rho$ : average mass density; $\rho_{\mathrm{kr}}$ : mass density required for a flat universe; $\tau$ : cosmic time; $\Omega: \rho / \rho_{\mathrm{kr}}$ (flat universe: $\Omega=1$ ).

\section{References}

1. Einstein A. Basics of the theory of relativity. 5th edition, Vieweg-Verlag 1982.

2. Einstein A. Cosmological considerations on general relativity. Session Reports of the Royal Prussian Academy of Sciences: Physical and Mathematical Class. 1917; 142-152.

3. Fölsing A. Albert Einstein, eine Biographie. Suhrkamp Verlag. 1993.

4. Genz H. wie die Zeit in die Wirklichkeit kam. Carl Hauser Verlag. 1999. ISBN 978-3-499-60731-8

5. Friedmann A. Die Welt als Raum und Zeit. Reprint of the original Russian edition of 1923. Verlag Harri Deutsch. 1923.

6. Greene B. The Fabric of the Cosmos: Time, Space, and the next Texture of Reality. Alfred A. Knopf publisher. 2004. ISBN: 0965900584

7. Prigogine I. Time, Chaos and the Quantum Towards the Resolution of the Time Paradox. Harmony Books, Crown Publishing. 1993.

8. Smolin L. Time Reborn - From the Crisis in Physics to the Future of the Universe. Houghton Mifflin Harcourt. 2013. ISBN: 9780547511726

9. Hawking S. A brief history of time. Bantam Dell Publishing Group. 1988 ISBN: 9780553173253

10. Kraus LM. A Universe from Nothing. Simon \& Schuster. 2013. ISBN 978-3-328-10309-7

11. Davies $P$. The Goldilocks enigma - why is the universe just right for life? Penguin Group. 2007. ISBN: 9780141901299

12. Fritsch H. Der Urknall - eine Fata Morgana. Shaker-Verlag. 2015.

13. Börner G. Kosmologie. Fischer Taschenbuch-Verlag. 2002.

14. Goenner $\mathrm{H}$. Einführung in die Kosmologie. Spektrum, Akademischer Verlag. 1994. ISBN 3-86025-332-8

15. Rauner M. Das moderne Bild vom Kosmos. GEOWISSEN Nr. 33, 2004.

16. Unzicker A. Vom Urknall zum Durchknall. Springer-Verlag Berlin. 2010. ISBN 978-3-642- 04836

17. Harrison ER. Cosmology: The Science of the universe. Cambridge university Press. 1981. ISBN 052166148 X 
18. Meschede D. Gerthsen Physik, 23rd Edition, Springer-Verlag. 2005.

19. Clark S. The Big Questions: The Universe. Spektrum, Akademischer Verlag. 2010.

20. Müller A. Raum und Zeit. Springer Spektrum. 2013. ISBN 978-3-82742858-5

21. Atkins R. Physics. John Wiley and Sons Inc. 1986. ISBN: 0471036196

22. Barrow JD. The World Within the World. Oxford university press. 1990. ISBN: 0192861085

23. Weber C, Kilian U. Lexikon der Physik. Spektrum Akademischer Verlag. 2004. ISBN: 3827413628
24. Mittelstaedt P. Der Zeitbegriff in der Physik. B.I.-Wissenschaftsverlag. 1989. ISBN 10: 3411031956

25. Penrose R. The Road to Reality. Jonathan Cape Random House. 2004. ISBN 0-224-04447-8

26. Prigogine I. From Being to Becoming - Time and Complexity in Physical Science. W.H.Freedman and company, San Francisco. 1980. ISBN: 9780716711087

27. Lorenzen D. SWR2 WISSEN, Radio broadcast from 23.12.2009.

28. Lorenzen D. SWR2 WISSEN, Radio broadcast from 28.01.2015.

29. Nicolai H. Alles nur Fassade, ZEIT-Interview with Hermann Nicolai, DIE ZEIT, 14.05.2005. 